\title{
Two- or Three-Dimensional Imagings of Simultaneous Visualization of Rat Pituitary Hormone and Its mRNA: Comparison between Electron Microscopy and Confocal Laser Scanning Microscopy with Semiconductor Nanocrystals (Quantum dots)
}

\author{
Akira Matsuno ${ }^{1}$, Johbu Itoh ${ }^{2}$, Susumu Takekoshi ${ }^{3}$, Tadashi Nagashima ${ }^{1}$ and \\ R. Yoshiyuki Osamura ${ }^{3}$ \\ ${ }^{1}$ Department of Neurosurgery, Teikyo University Ichihara Hospital, 3426-3 Anesaki, Ichihara City, Chiba 299-0111, \\ Japan, ${ }^{2}$ Teaching and Research Support Center, Tokai University School of Medicine, Boseidai, Isehara City, \\ Kanagawa 259-1100, Japan and ${ }^{3}$ Department of Pathology, Tokai University School of Medicine, Boseidai, \\ Isehara City, Kanagawa 259-1100, Japan
}

Received March 22, 2005; accepted June 13, 2005

Semiconductor nanocrystals (Quantum dots, Qdots) have recently been used in biological research, since they do not fade upon exposure to light, enabling us to obtain multicolor imaging due to a narrow emission peak that can be excited via a single wavelength of light. Utilizing the advantages of Qdot and confocal laser scanning microscopy (CLSM), we can obtain three-dimensional images of the intracellular localization of growth hormone and mRNA in a pituitary cell. The previous method of combined in situ hybridization (ISH) and immunohistochemistry (IHC) under electron microscopy has sufficient ultrastructural resolution, but can provide only twodimensional images. By contrast, ISH and IHC using Qdots and CLSM can optimally illustrate the simultaneous relationship between protein and mRNA in three dimensions. Such an approach enables us to better visualize functional images of proteins in relation to mRNA synthesis and localization.

Key words: in situ hybridization, immunohistochemistry, mRNA, Quantum dot, confocal laser scanning microscopy

\section{Introduction}

We have employed a combined electron microscopic (EM) in situ hybridization (ISH) and immunohistochemistry (IHC) (EM-ISH-IHC), in order to simultaneously investigate the intracellular localization of pituitary hormone and its mRNA [14-19, 23, 24]. The EM-ISH-IHC method is very useful for the study of the spatial relationship of mRNA and the encoded protein. This method has sufficiently high resolution for ultrastructure observation, but provides only a two-dimensional image of the mRNA and protein.

Correspondence to: Akira Matsuno, M.D., Ph.D., Department of Neurosurgery, Teikyo University Ichihara Hospital, 3426-3 Anesaki, Ichihara City, Chiba 299-0111, Japan.

E-mail: akirakun@med.teikyo-u.ac.jp
Confocal laser scanning microscopy (CLSM) combined with computed imaging analysis enables us to observe subcellular organelles, mRNA, and protein three-dimensionally in routinely processed light microscopic specimens [13, 9-12, 18, 22, 26-28, 30]. Meanwhile, semiconductor nanocrystals (Quantum dots, Qdots) that do not fade upon exposure to light and enable us to obtain multicolor images of molecules due to a narrow emission peak that can be excited via a single wavelength of light have recently been developed [4, 29]. Qdots have been used in biological research to detect signals of IHC and fluorescence in situ hybridization (FISH) [5-8, 25, 31]. Recently, we successfully applied the above-mentioned advantages of Qdots and CLSM to three-dimensional imagings of the intracellular localization of mRNA and protein [20]. In this paper, we compare the two methods, two-dimensional imagings of 
simultaneous visualization of rat growth hormone $(\mathrm{GH})$ and its mRNA using electron microscopy, and three-dimensional imagings using CLSM and nanocrystals (Qdots), and discuss their advantages and disadvantages.

\section{Combined ISH and IHC at an Electron Microscopic Level}

The detailed method of EM-ISH\&IHC was described in our previous reports [14-19]. Briefly, hybridization was carried out using biotinylated oligonucleotide probes. The hybridized signals of mRNA were detected with streptavidin-biotin complex (ABC) and horseradish peroxidase (HRP), and then developed with diaminobenzidine (DAB) and $\mathrm{H}_{2} \mathrm{O}_{2}$. After osmification and dehydration with ethanol, tissue sections were embedded in Epon resin. After polymerization, ultrathin sections were attached to Nickel grids. Subsequently, the immunoreactivity of the targeted protein was retrieved by $10 \% \mathrm{H}_{2} \mathrm{O}_{2}$ or with $4 \%$ sodium periodate. Immunohistochemical staining was carried out using primary antibody, and the immunoreaction was visualized with 20 $\mathrm{nm}$ protein A colloidal gold.

As shown in our previous reports [14-19], EM-ISH with an antisense probe for rat GH mRNA reveals its localization on the rough endoplasmic reticulum (RER) (Fig. 1). Subsequent immunohistochemical staining using anti-rat GH antibody and $20 \mathrm{~nm}$ protein A colloidal gold identifies rat GH mainly on the secretory granules and also in the cisternae of the RER (Fig. 1).

EM-ISH-IHC method has sufficient ultrastructural resolution to enable us to simultaneously visualize the intracellular localization of mRNA and encoded protein together

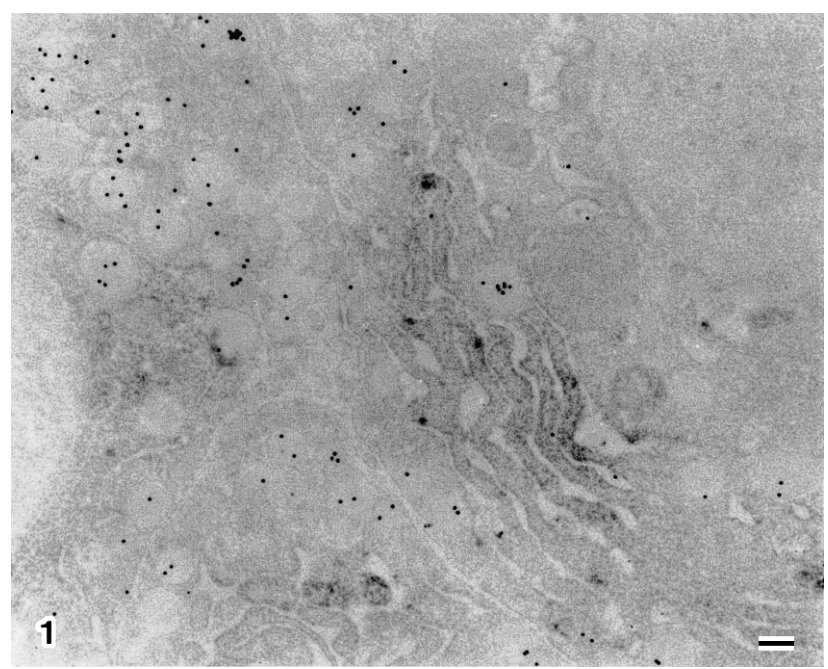

Fig. 1. EM-ISH with an antisense probe for rat GH mRNA reveals its localization on the rough endoplasmic reticulum (RER). Subsequent immunohistochemical staining using anti-rat GH antibody and $20 \mathrm{~nm}$ protein A colloidal gold identifies rat GH mainly on the secretory granules and also in the cisternae of the RER. (Cited from Ref. 16, Bar=200 nm) with subcellular organelles. However, the images are twodimensional.

\section{Combined ISH and IHC Using HRP-DAB for the Detection of mRNA and Qdot for the Detection of Protein}

The detailed method was described in our previous report [20]. Briefly, hybridization was carried out using biotinylated oligonucleotide probes. The hybridized signals of mRNA were detected with $\mathrm{ABC}$ and HRP, and then developed with DAB and $\mathrm{H}_{2} \mathrm{O}_{2}$. Subsequently, immunohistochemical staining was carried out using primary antibody, and the immunoreaction was visualized with Qdot.

Hybridization signals for rat GH mRNA were demonstrated under light microscopy using ABC-HRP. With the CLSM reflection mode, GH mRNA was observed as a DAB signal, and with the confocal mode, GH protein was observed as a $655 \mathrm{~nm}$ emission signal using Qdot 655 conjugated with anti-rabbit IgG (Quantum Dot Corp., Hayward, CA, USA) (Fig. 2).

\section{Combined ISH and IHC Using Qdots for the Detection of mRNA and Protein}

The detailed method was described in our previous report [20]. Briefly, hybridization was carried out using biotinylated oligonucleotide probes, and subsequently, immunohistochemical staining was carried out using primary antibody. Both the hybridized signals of mRNA and the immunopositive reactions were detected with different sized Qdots that had different emission peaks.

With the confocal mode, GH protein was observed as a $655 \mathrm{~nm}$ emission signal using using Qdot 655 conjugated with anti-rabbit IgG (Fig. 3), and GH mRNA was observed as a $605 \mathrm{~nm}$ emission signal using Qdot 605 conjugated with streptavidin (Quantum Dot Corp., Hayward, CA, USA) (Fig. $3)$. When GH mRNA and protein were located either in the same or adjacent places, their signals were detected in the mixed color image (Fig. 3). This finding was more prominently shown in the combined ISH\&IHC method using Qdots for the detection of mRNA and protein than in the combined ISH\&IHC method using HRP-DAB for the detec-

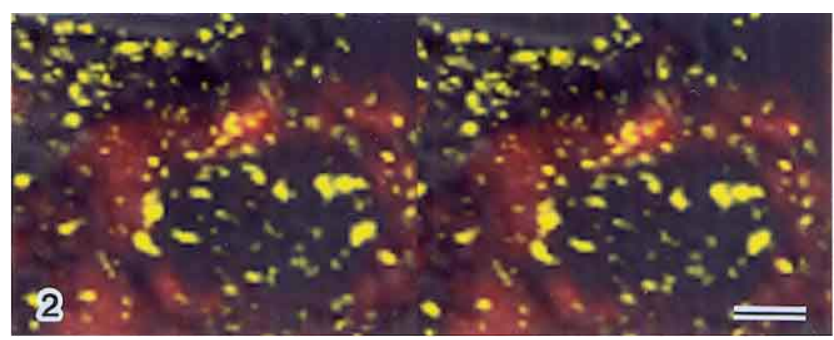

Fig. 2. With the CLSM reflection mode, GH mRNA was observed as a DAB signal (yellow), and with the confocal mode, GH protein was observed as a $655 \mathrm{~nm}$ emission signal using Qdot 655 (red). (stereo-images, Bar $=2 \mu \mathrm{m}$ ) 


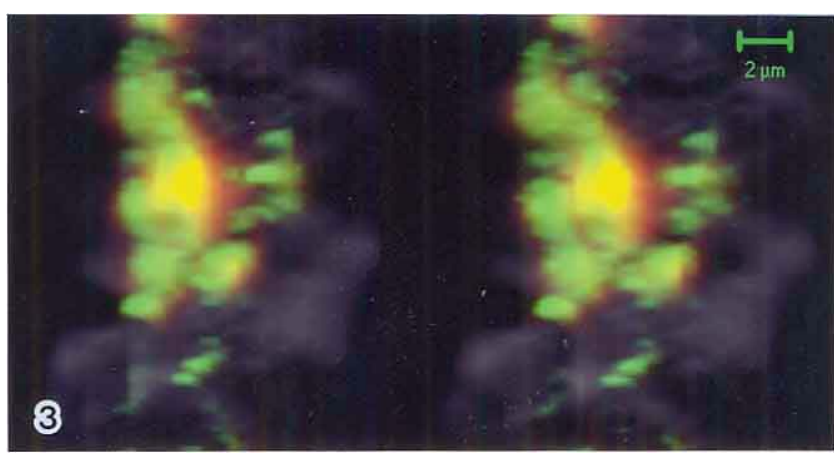

Fig. 3. With the confocal mode, GH protein was observed as a 655 $\mathrm{nm}$ emission signal using Qdot 655 (red), and GH mRNA was observed as a $605 \mathrm{~nm}$ emission signal using Qdot 605 (green). When GH mRNA and protein were located in the same or adjacent places, their signals were detected in the mixed color images (yellow). (stereo-images, Bar $=2 \mu \mathrm{m}$ )

tion of mRNA and Qdot for the detection of protein. The mixed color images of GH mRNA and protein conjugated with different sized Qdots mean that these molecules are located within the three-dimensional distance of $25 \pm 13 \mathrm{~nm}$ $[13,21]$.

\section{Discussion}

Qdots have narrow, symmetric emission spectra with multiple resolvable colors that can be excited simultaneously using a single excitation wavelength. The color of Qdots can be tuned to any chosen wavelength by simply changing their size. This property enables multiple labeling of subcellular molecules. Qdots have more stable signals than conventional fluorophores, such as FITC and Texas Red, and do not fade upon exposure to light. Xiao and Barker stated that significantly less signal loss was observed for Qdot probes than for FITC or Texas Red probes [31]. Furthermore, they found that Qdot signals were more than 11-fold stronger than those of fluorescein [31]. Due to their photostability and prominent signal intensity, Qdots have an advantage over conventional fluorophores.

Using these properties, we successfully visualized the intracellular localization of pituitary hormone and mRNA by using different sized Qdots with CLSM [20]. This analysis has several merits: it can be used with light microscopic specimens; it can be observed in any chosen cells and at any chosen depth of the section; it can be used to reconstruct three-dimensional images. Meanwhile, combined EMISH-IHC method provides higher resolutional images of subcellular organelles than combined ISH and IHC using Qdots and CLSM, and therefore, the EM-ISH-IHC method is essential for analyses at high resolution. However, the EM-ISH-IHC method has some limitations: it can be used only with EM specimens; it can observe only a small number of cells; and it provides only a two-dimensional image of mRNA and protein.

Intracellular organelles, such as RER and secretory granules, have a three-dimensional structure and localization. Three-dimensional images of the intracellular localization of mRNA and encoded protein enhance our understanding concerning the localization of mRNA and secreted protein. These observations were more prominently shown in combined ISH and IHC with Qdots for the detection of mRNA and protein than in combined ISH and IHC with HRP-DAB for the detection of mRNA and Qdot for the detection of protein. Two different sized Qdots can discriminate between two molecules that are located at a distance of more than $25 \pm 13 \mathrm{~nm}[13,21]$. Therefore, the mixed color images of GH mRNA and protein conjugated with different sized Qdots mean that these molecules are located within the three-dimensional distance of 12-38 nm.

By using ISH and IHC with Qdots and CLSM, one can optimize the simultaneous visualization of the relationship between protein and mRNA three-dimensionally. It thus enables us to better visualize the functional images of proteins in relation to mRNA synthesis and localization.

\section{References}

1. Arndt-Jovin, D. J., Robert-Nicoud, M., Kaufman, S. J. and Jovin, T. M. (1985) Fluorescence digital imaging microscopy in cell biology. Science 230; 247-256.

2. Arndt-Jovin, D. J., Robert-Nicoud, M. and Jovin, T. M. (1990) Probing DNA structure and function with a multi-wavelength fluorescence confocal laser microscope. J. Microsc. 157; 61-72.

3. Bauman, J. G., Bayer, J. A. and van Dekken, H. (1990) Fluorescent in-situ hybridization to detect cellular RNA by flow cytometry and confocal microscopy. J. Microsc. 157; 73-81.

4. Bruchez, M. Jr., Moronne, M., Gin, P., Weiss, S. and Alivisatos, A. P. (1998) Semiconductor nanocrystals as fluorescent biological labels. Science 281; 2013-2016.

5. Chan, W. C., Maxwell, D. J., Gao, X., Bailey, R. E., Han, M. and Nie, S. (2002) Luminescent quantum dots for multiplexed biological detection and imaging. Curr. Opin. Biotechnol. 13; $40-46$.

6. Gao, X., Chan, W. C. and Nie, S. (2002) Quantum-dot nanocrystals for ultrasensitive biological labeling and multicolor optical encoding. J. Biomed. Opt. 7; 532-537.

7. Gao, X. and Nie, S. (2003) Molecular profiling of single cells and tissue specimens with quantum dots. Trends Biotechnol. 21;371373.

8. Han, M., Gao, X., Su, J. Z. and Nie, S. (2001) Quantum-dottagged microbeads for multiplexed optical coding of biomolecules. Nat. Biotechnol. 19; 631-635.

9. Hozak, P., Novak, J. T. and Smetana, K. (1989) Three-dimensional reconstructions of nucleolus-organizing regions in PHAstimulated human lymphocytes. Biol. Cell 66; 225-233.

10. Itoh, J., Osamura, R. Y. and Watanabe, K. (1992) Subcellular visualization of light microscopic specimens by laser scanning microscopy and computer analysis: a new application of image analysis. J. Histochem. Cytochem. 40; 955-967.

11. Itoh, J., Sanno, N., Matsuno, A., Itoh, Y., Watanabe, K. and Osamura, R. Y. (1997) Application of confocal laser scanning microscopy (CLSM) to visualize prolactin (PRL) and PRL mRNA in the normal and estrogen-treated rat pituitary glands using non-fluorescent probes. Microsc. Res. Tech. 39; 157-167.

12. Itoh, J., Matsuno, A., Yamamoto, Y., Kawai, K., Serizawa, A., Watanabe, K., Itoh, Y. and Osamura, R. Y. (2001) Confocal laser scanning microscopic imaging of subcellular organelles, mRNA, protein products, and the microvessel environment. Acta 
Histochem. Cytochem. 34; 285-297.

13. Lacoste, T. D., Michalet, X., Pinaud, F., Chemla, D. S., Alivisatos, A. P. and Weiss, S. (2000) Ultrahigh-resolution multicolor colocalization of single fluorescent probes. Proc. Natl. Acad. Sci. U S A 97; 9461-9466.

14. Matsuno, A., Utsunomiya, H., Ohsugi, Y., Takekoshi, S., Sanno, N., Osamura, R. Y., Nagao, K., Tamura, A. and Nagashima, T. (1996) Simultaneous ultrastructural identification of growth hormone and its messenger ribonucleic acid using combined immunohistochemistry and non-radioisotopic in situ hybridization: a technical note. Histochem. J. 28; 703-707.

15. Matsuno, A., Ohsugi, Y., Utsunomiya, H., Takekoshi, S., Munakata, S., Nagao, K., Osamura, R. Y., Tamura, A. and Nagashima, T. (1998) An improved ultrastructural doublestaining method of rat growth hormone and its mRNA using LR White resin: a technical note. Histochem. J. 30; 105-109.

16. Matsuno, A., Nagashima, T., Osamura, R. Y. and Watanabe, K. (1998) Application of ultrastructural in situ hybridization combined with immunohistochemistry to pathophysiological studies of pituitary cell: Technical Review. Acta Histochem. Cytochem. $31 ; 259-265$.

17. Matsuno, A., Nagashima, T., Takekoshi, S., Utsunomiya, H., Sanno, N., Osamura, R. Y., Watanabe, K., Tamura, A. and Teramoto, A. (1998) Ultrastructural simultaneous identification of growth hormone and its messenger ribonucleic acid. Endocr. J. 45[Suppl]; S101-S104.

18. Matsuno, A., Itoh, J., Osamura, R. Y., Watanabe, K. and Nagashima, T. (1999) Electron microscopic and confocal laser scanning microscopic observation of subcellular organelles and pituitary hormone mRNA: application of ultrastructural in situ hybridization and immunohistochemistry to the pathophysiological studies of pituitary cells. Endocr. Pathol. 10; 199-211.

19. Matsuno, A., Nagashima, T., Ohsugi, Y., Utsunomiya, H., Takekoshi, S., Munakata, S., Nagao, K., Osamura, R. Y. and Watanabe, K. (2000) Electron microscopic observation of intracellular expression of mRNA and its protein product: Technical review on ultrastructural in situ hybridization and its combination with immunohistochemistry. Histol. Histopathol. 15; 261-268

20. Matsuno, A., Itoh, J., Takekoshi, S., Nagashima, T. and Osamura, R. Y. (2005) Three-dimensional imagings of the intracellular localization of growth hormone and prolactin and their mRNA using nanocrystal (Quantum dot) and confocal laser scanning microscopy techniques. J. Histochem. Cytochem. 53; 833-838.

21. Michalet, X., Pinaud, F., Lacoste, T. D., Dahan, M., Bruchez, M. P., Alivisatos, A. P. and Weiss, S. (2001) Properties of fluorescent semiconductor nanocrystals and their application to biological labeling. Single Mol. 4; 261-276.

22. Michel, E. and Parsons, J. A. (1990) Histochemical and immunocytochemical localization of prolactin receptors on $\mathrm{Nb} 2$ lymphoma cells: applications of confocal microscopy. J. Histochem. Cytochem. 38; 965-973.

23. Osamura, R. Y., Itoh, Y. and Matsuno, A. (2000) Application of plastic embedding to electron microscopic immunocytochemistry and in situ hybridization in observations of production and secretion of peptide hormones. J. Histochem. Cytochem. 48; 885-891.

24. Osamura, R. Y., Tahara, S., Kurotani, R., Sanno, N., Matsuno, A. and Teramoto, A. (2000) Contributions of immunohistochemistry and in situ hybridization to the functional analysis of pituitary adenomas. J. Histochem. Cytochem. 48; 445-458.

25. Pathak, S., Choi, S. K., Arnheim, N. and Thompson, M. E. (2001) Hydroxylated quantum dots as luminescent probes for in situ hybridization. J. Am. Chem. Soc. 123; 4103-4104.

26. Robinson, J. M. and Batten, B. E. (1989) Detection of diaminobenzidine reactions using scanning laser confocal reflectance microscopy. J. Histochem. Cytochem. 37; 1761-1765.

27. Takamatsu, T. and Fujita, S. (1988) Microscopic tomography by laser scanning microscopy and its three-dimensional reconstruction. J. Microsc. 149; 167-174.

28. Tao, W., Walter, R. J. and Berns, M. W. (1988) Laser-transected microtubules exhibit individuality of regrowth, however most free new ends of the microtubules are stable. J. Cell Biol. 107; $1025-1035$.

29. Wang, C., Shim, M. and Guyot-Sionnest, P. (2001) Electrochromic nanocrystal quantum dots. Science 291; 2390-2392.

30. White, J. G., Amos, W. B. and Fordham, M. (1987) An evaluation of confocal versus conventional imaging of biological structures by fluorescence light microscopy. J. Cell Biol. 105; 41-48.

31. Xiao, Y. and Barker, P. E. (2004) Semiconductor nanocrystal probes for human metaphase chromosomes. Nucleic Acids Res. 32; e28. 